\title{
A Nonlinear Second-order Spacecraft Attitude Tracking Control Model for Control System Stabilization
}

\author{
Xiaoyi Wang ${ }^{1}$ \\ ${ }^{1}$ Center for Control Theory and Guidance Technology, Harbin Institute of Technology, Harbin, China
}

\begin{abstract}
A control model for the direct parameter approach for spacecraft attitude tracking is presented in this paper. First of all, the spacecraft attitude tracking control model is built up by the error equation of the second-order nonlinear quaternion-based attitude system. A problem of control system stabilization is raised based on the control model. Compared with other control models, the second-order can offer the advantages of no- approximation and clear control states. The basic spacecraft control model has to focus on to the two variables which are angular rate and attitude quaternion, however, the new attitude control problem is only with respect to one variable which is the spacecraft attitude quaternion. Therefore, the second-order model is simpler and clear than basic first-order model. Keywords: spacecraft attitude tracking; nonlinear system; quaternion representation; second-order control model
\end{abstract}

\section{Introduction}

As a significant part of spacecraft navigation control, spacecraft attitude control has been a hot problem for several years. Many different methods are applied on the problem of spacecraft attitude control as in [1-6] and [9-11]. For instance, Bang designed a new sliding model control method which can deal with flexible spacecraft attitude maneuver problem in [5]. Singh utilized adaptive output feedback control method to track the flexible spacecraft attitude in [6]. Additionally, Guan raised a direct parametric approach to stabilize the nonlinear second-order spacecraft attitude model, and the controller can turn the original model into a stable linear constant system certain degrees of freedom in [1].

According to the research background of spacecraft attitude control, it is suggested that spacecraft attitude tracking needs further research and has more profound applications. Compared with Euler representation, quaternion representation can describe large-angle attitude tracking without singular points which should appear when the changing of Euler angle is bigger than $90^{\circ}$. Moreover, this paper raises a second-order system based on the spacecraft attitude dynamical and kinematical differential equations. Although most of methods would like to deal with the spacecraft attitude control problem by first-order models, this paper takes advantage of the second-order model. Furthermore, the second-order control model is with no-approximation, which means it can precisely describe the process of spacecraft attitude tracking. Moreover, the second-order model is only with respect one control variable which is simpler than first-order model with two control variables. In addition, the second-order model has broad application prospects, for instance, Duan offered a direct parametric approach which can turn a nonlinear second-order system into a stable linear constant system by the parametric controller as in [1-3], and [7-8].

\section{Basic spacecraft attitude tracking problem}

According to the attitude dynamics and kinematics of a rigid spacecraft in the inertial system, the error attitude dynamics of a rigid spacecraft relative to a mobile tracking target in the inertial system is given by

$$
\begin{aligned}
J \dot{\omega}_{e} & =-\omega \times J \omega \\
& +J\left(\omega \times C\left(q_{e}\right) \omega_{r}-C\left(q_{e}\right) \dot{\omega}_{r}\right), \\
& +u+d
\end{aligned}
$$

where

$$
J=\operatorname{diag}\left(J_{x}, J_{y}, J_{z}\right)
$$

is the rotating inertia matrix of the spacecraft,

$\omega$ is the angular rate vector, $\omega_{e}$ is the error angular rate vector relative to the mobile tracking target, $\omega_{r}$ is the angular rate vector for target, $\mathrm{u}$ is the input control torque vector, and $\mathrm{d}$ is the disturbance torque vector. Besides, $\omega$, $\omega_{e}, \omega_{r}, u$ and $d$ belong to $\mathbf{R}^{3 \times 1}$.In addition, the $\mathbf{C}\left(q_{e}\right)$ is the transition matrix and its expression is

$$
C\left(q_{e}\right)=\left[\begin{array}{lll}
C_{1}\left(q_{e}\right) & C_{2}\left(q_{e}\right) & C_{3}\left(q_{e}\right)
\end{array}\right],
$$

where 


$$
\begin{gathered}
C_{1}\left(q_{e}\right)=\left[\begin{array}{c}
q_{e 1}^{2}-q_{e 2}^{2}-q_{e 3}^{2}+q_{e 0}^{2} \\
2\left(q_{e 1} q_{e 2}-q_{e 3} q_{e 0}\right) \\
2\left(q_{e 1} q_{e 3}+q_{e 2} q_{e 0}\right)
\end{array}\right], \\
C_{2}\left(q_{e}\right)=\left[\begin{array}{c}
2\left(q_{e 1} q_{e 2}+q_{e 3} q_{e 0}\right) \\
-q_{e 1}^{2}+q_{e 2}^{2}-q_{e 3}^{2}+q_{e 0}^{2} \\
2\left(q_{e 2} q_{e 3}-q_{e 1} q_{e 0}\right)
\end{array}\right], \\
C_{3}\left(q_{e}\right)=\left[\begin{array}{c}
2\left(q_{e 1} q_{e 3}-q_{e 2} q_{e 0}\right) \\
2\left(q_{e 2} q_{e 3}+q_{e 1} q_{e 0}\right) \\
-q_{e 1}^{2}-q_{e 2}^{2}+q_{e 3}^{2}+q_{e 0}^{2}
\end{array}\right],
\end{gathered}
$$

and

$$
q_{e}=\left[\begin{array}{llll}
q_{e 0} & q_{e 1} & q_{e 2} & q_{e 3}
\end{array}\right]^{T} .
$$

The $\omega_{e}$ can be expressed by

$$
\omega_{e}=\omega-C(e) \omega_{r} .
$$

Then the kinematics of a rigid spacecraft in the inertial system based on error quaternion is given by

$$
\left[\begin{array}{c}
\dot{q}_{e 0} \\
\dot{q}_{e 1} \\
\dot{q}_{e 2} \\
\dot{q}_{e 3}
\end{array}\right]=\frac{1}{2}\left[\begin{array}{ccc}
-q_{e 1} & -q_{e 2} & -q_{e 3} \\
q_{e 0} & -q_{e 3} & q_{e 2} \\
q_{e 3} & q_{e 0} & -q_{e 1} \\
-q_{e 2} & q_{e 1} & q_{e 0}
\end{array}\right]\left[\begin{array}{c}
\omega_{e x} \\
\omega_{e y} \\
\omega_{e z}
\end{array}\right],
$$

where $\omega_{e}$ is the error angular rate vector relative to the mobile tracking target, and $q_{e}$ is the error quaternion relative to the target.

To solve the attitude problem, a suitable controller $u$ is given by

$$
u=f\left(\omega_{e}, q_{e 0}, q_{e 1}, q_{e 2}, q_{e 3}\right),
$$

which can make the close-loop spacecraft attitude system stable.

When the system is stable, which means

$$
\left\{\begin{array}{l}
\lim _{t \rightarrow \infty} \omega_{e}=0, \lim _{t \rightarrow \infty} q_{e 0}=1, \lim _{t \rightarrow \infty} q_{e 1}=0 \\
\lim _{t \rightarrow \infty} q_{e 2}=0, \lim _{t \rightarrow \infty} q_{e 3}=0
\end{array},\right.
$$

the spacecraft will successfully achieves the attitude tracking goal.

\section{Spacecraft attitude tracking problem based on the second-order control problem}

In this section, a second-order spacecraft attitude control model is built by attitude dynamical equations and kinematical equations. Additionally, that model is described by quaternion and without any approximation. The problem of quaternion-based spacecraft attitude tracking is raised based on the attitude control model.

Generally, the error quaternion $q_{e}$ has the constraint which is

$$
q_{e 0}{ }^{2}+q_{e 1}^{2}+q_{e 2}^{2}+q_{e 3}^{2}=1 .
$$

Based on the (12) a simple variable $e$ can be expressed as

$$
e=\left[\begin{array}{lll}
e_{1} & e_{2} & e_{3}
\end{array}\right]^{T}=\left[\begin{array}{lll}
q_{e 1} & q_{e 2} & q_{e 3}
\end{array}\right]^{T} .
$$

Additionally, the $q_{e 0}$ can be denoted as

$$
q_{e 0}=e_{0}=\sqrt{1-\left(e_{1}^{2}+e_{2}^{2}+e_{3}^{2}\right)} .
$$

With the expression of $e$, the (5) can be written as

$$
\dot{e}=\frac{1}{2} T(e) \omega_{e}=\frac{1}{2}\left[\begin{array}{ccc}
e_{0} & -e_{3} & e_{2} \\
e_{3} & e_{0} & -e_{1} \\
-e_{2} & e_{1} & e_{0}
\end{array}\right] \omega_{e} .
$$

Taking derivative of the equation (12) with respect to time $t$, it yields

$$
\ddot{e}=\frac{1}{2} \frac{d T(e)}{d t} \omega_{e}+\frac{1}{2} T(e) \dot{\omega}_{e} .
$$

The expression for $\omega_{e}$ can be derived from the (12), it yields

$$
\begin{aligned}
\omega_{e} & =2\left(I_{3}-e e^{T}\right)^{-1} T^{T}(e) \dot{e} \\
& =2 \Phi(e) T^{T}(e) \dot{e}
\end{aligned}
$$

where

$$
\Phi(e)=\left(I_{3}-e e^{T}\right)^{-1}=\left[\begin{array}{c}
\Phi_{1}(e) \\
\Phi_{2}(e) \\
\Phi_{3}(e)
\end{array}\right] .
$$

Thus the variables of the $\omega_{e}$ can be expressed as

$$
\left\{\begin{array}{l}
\omega_{e x}=2 \Phi_{1}(e) T^{T}(e) \dot{e} \\
\omega_{e y}=2 \Phi_{2}(e) T^{T}(e) \dot{e} . \\
\omega_{e z}=2 \Phi_{3}(e) T^{T}(e) \dot{e}
\end{array}\right.
$$

The next step is to summarize the second-order spacecraft control model through the (1) and (16). First of all, the expression for $\dot{\omega}_{e}$ can be derived by (1) as

$$
\begin{aligned}
\dot{\omega}_{e} & =J^{-1}\left[\omega_{e} \times\left(J \omega_{e}\right)\right] \\
& -J^{-1}\left\{\omega_{e} \times\left[J C(e) \omega_{r}\right]\right\} \\
& -J^{-1}\left\{\left[C(e) \omega_{r}\right] \times\left[J \omega_{e}\right]\right\} \\
& -J^{-1}\left\{\left[C(e) \omega_{r}\right] \times\left[J C(e) \omega_{r}\right]\right\} \\
& +\left[\omega_{e} \times\left(C(e) \omega_{r}\right)\right] \\
& -C(e) \dot{\omega}_{r}+J^{-1}(u+d)
\end{aligned}
$$

When these expressions are denoted as,

$$
\begin{aligned}
& \Gamma(J, \mathrm{e}, \dot{\mathrm{e}})=\left[\begin{array}{ccc}
0 & 0 & \Gamma_{c} \\
\Gamma_{a} & 0 & 0 \\
0 & \Gamma_{b} & 0
\end{array}\right], \\
& \left\{\begin{array}{l}
\Gamma_{a}=\frac{J_{z}-J_{x}}{J_{y}} \Phi_{3}(e) T^{T}(e) \dot{e} \\
\Gamma_{b}=\frac{J_{x}-J_{y}}{J_{z}} \Phi_{1}(e) T^{T}(e) \dot{e}, \\
\Gamma_{c}=\frac{J_{y}-J_{z}}{J_{x}} \Phi_{2}(e) T^{T}(e) \dot{e}
\end{array}\right. \\
& G=\left[\begin{array}{l}
G_{1} \\
G_{2} \\
G_{3}
\end{array}\right]=\left[\begin{array}{l}
{\left[J C(e) \omega_{r}\right]_{x}} \\
{\left[J C(e) \omega_{r}\right]_{y}} \\
{\left[J C(e) \omega_{r}\right]_{z}}
\end{array}\right],
\end{aligned}
$$




$$
H=\left[\begin{array}{l}
H_{1} \\
H_{2} \\
H_{3}
\end{array}\right]=\left[\begin{array}{l}
{\left[C(e) \omega_{r}\right]_{x}} \\
{\left[C(e) \omega_{r}\right]_{y}} \\
{\left[C(e) \omega_{r}\right]_{z}}
\end{array}\right] .
$$

and the model can be written as

$$
\begin{aligned}
\ddot{e} & =\left(\begin{array}{c}
\frac{d T}{d t} \Phi(e)+2 T \Gamma(J, e, \dot{e}) \Phi(e) \\
-T\left[\begin{array}{c}
H_{2} \Phi_{3}-H_{3} \Phi_{2} \\
H_{3} \Phi_{1}-H_{1} \Phi_{3} \\
H_{1} \Phi_{2}-H_{2} \Phi_{1}
\end{array}\right] \\
+J^{-1} T\left[\begin{array}{c}
H_{2} J_{z} \Phi_{3}-H_{3} J_{y} \Phi_{2} \\
H_{3} J_{x} \Phi_{1}-H_{1} J_{z} \Phi_{3} \\
H_{1} J_{y} \Phi_{2}-H_{2} J_{x} \Phi_{1}
\end{array}\right] \\
+J^{-1} T\left[\begin{array}{l}
G_{3} \Phi_{2}-G_{2} \Phi_{3} \\
G_{1} \Phi_{3}-G_{3} \Phi_{1} \\
G_{2} \Phi_{1}-G_{1} \Phi_{2}
\end{array}\right]
\end{array}\right) T^{T} \dot{e} \\
& -\frac{1}{2} T\left\{J^{-1}[H \times(J H)]+C(e) \dot{\omega}_{r}\right\} \\
& +\frac{1}{2} T J^{-1}(u+d)
\end{aligned}
$$

In addition, the spacecraft attitude tracking model (25) can be written in the second-order form as

$$
\begin{aligned}
& A_{2}(\theta, e, \dot{e}) \ddot{e}+A_{1}(\theta, e, \dot{e}) \dot{e} \\
& +A_{0}(\theta, e, \dot{e}) e+\xi(\theta, e, \dot{e}, t)=B(\theta, e, \dot{e}) u
\end{aligned}
$$

where

$$
A_{1}(\theta, e, \dot{e})=-\left(\begin{array}{c}
A_{2}(\theta, e, \dot{e})=I_{3}, \\
\left.\frac{d T}{d t} \Phi(e)+2 T \Gamma(J, e, \dot{e}) \Phi(e)\right) \\
-T\left[\begin{array}{l}
H_{2} \Phi_{3}-H_{3} \Phi_{2} \\
H_{3} \Phi_{1}-H_{1} \Phi_{3} \\
H_{1} \Phi_{2}-H_{2} \Phi_{1}
\end{array}\right] \\
{\left[\begin{array}{l}
H_{2} J_{z} \Phi_{3}-H_{3} J_{y} \Phi_{2} \\
H_{3} J_{x} \Phi_{1}-H_{1} J_{z} \Phi_{3} \\
H_{1} J_{y} \Phi_{2}-H_{2} J_{x} \Phi_{1}
\end{array}\right]} \\
+\left[\begin{array}{l}
G_{3} \Phi_{2}-G_{2} \Phi_{3} \\
G_{1} \Phi_{3}-G_{3} \Phi_{1} \\
G_{2} \Phi_{1}-G_{1} \Phi_{2}
\end{array}\right] \\
A_{0}(\theta, e, \dot{e})=0_{3}, \\
B(\theta, e, \dot{e})=\frac{1}{2} T(e) J^{-1}, \\
\xi(\theta, e, \dot{e}, t)=\frac{1}{2} T\left\{\begin{array}{l}
J^{-1}[H \times(J H)] \\
+C(e) \dot{\omega}_{r} \\
-J^{-1} d
\end{array}\right\}
\end{array}\right\}
$$

To solve the second-order spacecraft attitude tracking problem defined by (25), a suitable controller $u$ is given by

$$
u=f\left(e_{1}, e_{2}, e_{3}, t\right),
$$

which can make the close-loop spacecraft attitude system stable.
When the system is stable, which means

$$
\left\{\begin{array}{l}
\lim _{t \rightarrow \infty} e_{1}=0, \lim _{t \rightarrow \infty} e_{2}=0, \lim _{t \rightarrow \infty} e_{3}=0 \\
\lim _{t \rightarrow \infty} \dot{e}_{1}=0, \lim _{t \rightarrow \infty} \dot{e}_{2}=0, \lim _{t \rightarrow \infty} \dot{e}_{3}=0
\end{array},\right.
$$

the spacecraft will successfully achieves the attitude tracking goal.

\section{Conclusion}

A control model is built by the error attitude dynamics and attitude kinematics for spacecraft in this paper. This model is based on the quaternion representation which can be suitable for the spacecraft large attitude angular tracking. Additionally, this model is a nonlinear second-order no-approximate attitude system. As mentioned earlier, the control model can precisely describe the error attitude of spacecraft relative to mobile target in the space. Compared with the first-order model, the second-order model is only with respect one variable which is the spacecraft attitude quaternion. Thus, the second-order model can be considered simpler and clearer than first-order model. Finally, the model is expressed in the general second-order quasi-linear system form, which has profound application prospects on nonlinear control and linear control methods.

\section{Acknowledgement}

I am greatly indebted to my supervisor, Professor Guangren Duan, for his valuable instructions and suggestions on my paper and thesis, as well as his beneficial approach provides the theory basis for this paper.

\section{Reference}

1. Duan, Guang Ren. Quaternion-based satellite attitude control-A direct parametric approach. International Conference on Control, Automation \& Systems IEEE, pp. 935-941, (2014)

2. Duan, Guang Ren. Satellite attitude control-A direct parametric approach. Intelligent Control and Automation IEEE,,pp. 3989 - 3996, (2014)

3. Duan, Guang Ren. Direct parametric control of fully-actuated second-order nonlinear systems-The normal case. 2014 China Control Conference (CCC) 33rd Chinese IEEE, pp. 2406-2413, (2014)

4. Tewari,Ashish. Spacecraft Attitude Control.Aerospace Technologies Advancements. pp. 357-390, (2010)

5. Bang, Hyochoong, C. K. Ha, and H. K. Jin. Flexible spacecraft attitude maneuver by application of sliding mode control. Acta Astronautica, pp. 841-850, (2005).

6. Singh, Sahjendra N., and R. Zhang. Adaptive output feedback control of spacecraft with flexible appendages by modeling error compensation. Acta Astronautica, 54, pp. 229-243,(2004)

7. Duan, Guang Ren, and B. Zhou. Solution to the second-order Sylvester matrix equation 
$\mathrm{MVF} 2+\mathrm{DVF}+\mathrm{KV}=\mathrm{BW}$. IEEE Transactions on Automatic Control. 51, pp. 805-809, (2006)

8. Duan, Guang Ren. Generalized Sylvester Equations: Unified Parametric Solutions. Crc Press, (2015)

9. Gao, Chunyan, Q. Zhao, and G. Duan. Robust actuator fault diagnosis scheme for satellite attitude control systems. Journal of the Franklin Institute, 9, pp. 2560-2580, (2013)

10. Lu, Kunfeng, and Y. Xia. Adaptive attitude tracking control for rigid spacecraft with finite-time convergence. Automatica. 49, pp. 3591-3599, (2013)

11. Yoon, Hyungjoo, and P. Tsiotras. Spacecraft Adaptive Attitude and Power Tracking with Variable Speed Control Moment Gyroscopes. Journal of Guidance Control \& Dynamics . 25, pp. 1081-1090, (2002) 